\title{
Pharmacology and Metabolism of Voriconazole and Posaconazole in the Treatment of Invasive Aspergillosis - REVIEW OF THE LITERATURE
}

\author{
M. Sandherr ${ }^{1}$, G. Maschmeyer ${ }^{2}$ \\ ${ }^{1}$ Hematology and Oncology Specialized Practice, Weilheim, Germany \\ ${ }^{2}$ Klinikum Ernst von Bergmann, Dept. of Hematology, Oncology and Palliative Care, Potsdam, Germany
}

\begin{abstract}
Invasive fungal infections, predominantly aspergillosis and candidiasis, are among the most important causes of morbidity and mortality in immunocompromised patients. Primarily, patients with acute leukemia undergoing myelosuppressive chemotherapy and allogeneic stem cell transplant recipients are affected. Up to $60 \%$ of patients with invasive aspergillosis, the most common invasive mycosis among patients with hematologic malignancies, may still die of their infection, once it has become clinically overt. The spectrum of antifungal agents for clinical use now has expanded over the past ten years and includes the novel class of the echinocandins and two newer generation triazoles with an extended spectrum of activity against a wide range of fungal pathogens. This review will address pharmacological characteristics of the two broad-spectrum antifungal azoles, voriconazole and Posaconazole, which are important for their proper use in clinical practice.
\end{abstract}

\section{INTRODUCTION}

The incidence of invasive fungal infections has increased over the past decades. This is due to an increased number of at-risk immunocompromised individuals, such as patients with acute leukemia undergoing myelosuppressive chemotherapy or allogeneic stem cell transplantation. Advances in treatment options for hematologic malignancies, severe graft-versus-host disease and serious bacterial infections as well as less myelosuppressive conditioning regimens for allogeneic stem cell transplantation have improved clinical outcome for patients, resulting in an increasing number of patients living with a profoundly compromised immune system. One of the sequelae is the rise of invasive fungal diseases (IFD), which for instance have doubled in the past twenty years among allogeneic stem cell transplant recipients [1]. Besides relapse of leukemia and severe bacterial infection, IFD remains the main risk factor for poor outcome of leukemia treatment [2]. Up to $60 \%$ of patients with invasive aspergillosis, representing the most common invasive mycosis among patients with hematologic malignancies may still die of their infection, once it has become clinically overt [3]. This has remained a challenge over the past decades, while the spectrum of antifungal agents available for clinical use now has expanded markedly. Therapeutic options now include the novel class of the echinocandins and two newer generation triazoles with an extended spectrum of activity against a wide range of fungal pathogens [4].

However, many of the new antifungal agents have a pronounced variability in drug levels based on either inconsistent absorption or elimination, resulting in a wide interpatient variability of serum concentrations. Therefore, treatment with the most potent and safe antifungal agent in the most appropriate dosage is one of the main challenges for the clinical management of invasive fungal diseases.

Azole antifungal drugs are frequently used for prophylaxis and treatment of IFD [5]. They exhibit a large number of drug-drug interactions. To date, fluconazole, itraconazole, voriconazole and posaconazole are used for prophylaxis and treatment of IFD in patients with hematologic malignancies. They are substrates for and inhibitors of cytochrome P450 (CYP450) isoenzymes, as well as inhibitors of membrane transporters such as p-glycoprotein (P-gP). The antifungal triazoles target ergosterol biosynthesis by inhibiting the fungal cytochrome P450-dependent enzyme lanosterol 14-alpha-demethylase, causing cell membrane defects and cell death or inhibition of cell growth and replication. The triazoles also inhibit cytochrome P450-dependent enzymes of the functional respiration chain. Itraconazole, voriconazole and posaconazole are active in vitro and in vivo against all common species of Aspergillus. So far, clinically relevant resistance has only rarely been reported, but may become increasingly important in the future [6].

This review will focus on pharmacology and metabolism of voriconazole and posaconazole, the most important antifungal azoles used in clinical hematology to date. Pharmacokinetic and pharmacodynamic aspects, which are clinically relevant for establishing optimal dosing regimens against invasive fungal pathogens, will be described with special emphasis on metabolism and efficacy of the drugs in patients with hematologic malignancies and hematopoietic stem cell transplant recipients (Table 1 ).

\section{VORICONAZOLE}

Voriconazole is a second-generation triazole with broad spectrum of antifungal activity. The most important therapeutic impact is related to its activity against all common Aspergillus spp. It is considered the first-line drug for the treatment of invasive aspergillosis [7]. Voriconazole is also approved for treatment of candidemia 
Table 1. Pharmacokinetic properties of voriconazole and posaconazole.

\begin{tabular}{lll}
\hline Property & Posaconazole & Voriconazole \\
\hline Bioavailability & $\begin{array}{l}\text { variable } \\
\text { (depending on dosage regimen }\end{array}$ & $>95 \%$ \\
and food) & \\
Protein binding & $>98 \%$ & $58 \%$ \\
$\mathrm{Vd} / \mathrm{F}(\mathrm{L} / \mathrm{kg})$ & $7-25$ & 4.6 \\
$\mathrm{~T}_{\max }(\mathrm{h})$ & $3-6$ & $1-2$ \\
Metabolism & hepatic: glucuronidation & hepatic: CYP2C19, 2C9, 3 A4 \\
$\mathrm{t}_{1 / 2}(\mathrm{~h})$ & $15-35$ & $6-24$ \\
Elimination route & $<1 \%$ excreted unchanged in urine & hepatic; $<2 \%$ excreted \\
& $66 \%$ excreted unchanged in faeces & unchanged in urine \\
\hline
\end{tabular}

$\mathrm{T}_{1 / 2}$ : elimination half life; $\mathrm{t}_{\max }$ : time to reach the maximum plasma concentration; Vd/F: apparent volume of distribution after oral administration

in non-neutropenic patients, esophageal candidiasis and disseminated candidiasis and as first-line therapy for mycoses caused by Scedosporium and Fusarium species. It is not active against mucormycoses, primarily caused by Rhizopos, Mucor and Absidia species.

\section{PHARMACOKINETICS}

Pharmacokinetics describes and predicts the time course of drug concentrations in body fluids. Voriconazole has non-linear pharmacokinetics and its dose-response relationship exhibits wide interpatient variability. The therapeutic index is narrow, and serum concentrations are significantly influenced by a broad range of drug-drug interactions.

Voriconazole can be given orally or as an intravenous infusion. After oral administration, it is absorbed rapidly within 2 hours with an oral bioavailability of $>90 \%$ [8]. Absorption is not affected by gastric $\mathrm{pH}$, but food delays absorption and reduces bioavailability by $22 \%$. Therefore, voriconazole should be taken on an empty stomach [9]. Oral voriconazole reaches steady-state concentrations after 5-7 days of multiple oral administrations depending on the dosage regimen. The time to steady-state can be reduced to 1-2 days by starting with a loading dose. Studies showed non-linear pharmacokinetics with $\mathrm{Cmax}$ and area under the plasma concentration time curve (AUC) increasing disproportionately with dose for both intravenous and oral application of the drug [10]. This indicates saturation of its metabolism with respect to dose. The variability in plasma concentration and systemic dosing depends on genetic polymorphisms of the cytochrome P450 (CYP) isoenzymes, which are involved in the metabolism of voriconazole.

It is a major substrate for the CYP2C19 isoenzyme [11]. Depending on genetic polymorphisms, resulting in poor metabolizers and extensive (or rapid) metabolizers, significantly different plasma concentrations are observed despite identical dosing schedules, and low trough concentrations have been reported to be associated with poor clinical results. This has been documented in healthy volunteers, in patients with IFD and those undergoing hematopoietic stem cell transplantation $[12,13,14]$. In patients receiving voriconazole for antifungal prophylaxis, $15 \%$ had undetectable concen- trations and $27 \%$ had trough levels below $0.5 \mathrm{mg} / \mathrm{l}$. Only $62 \%$ of stem cell transplant recipients had therapeutic concentrations between 0.5 and $2 \mathrm{mg} / 1$ [15]. In one study examining the pharmacokinetics of voriconazole in patients after stem cell transplantation, a wide range of serum concentrations even in intraindividual measurements was observed [13].

Plasma protein binding of voriconazole is $58 \%$ and is independent of plasma concentration. Cerebrospinal fluid concentrations of voriconazole are $50 \%$ of plasma concentrations with even higher concentrations obtained in brain tissue. According to the "free-drug hypothesis" of anti-infective agents, the free-drug concentration must be considered when exploring the relationship between pharmacokinetic parameters and in vivo activity [16]. The mean elimination half-life (t1/2) of voriconazole is about 6 hours [17]. The mean $t 1 / 2$ values were greater after multiple oral or intravenous administrations. Voriconazole is distributed widely into tissues with a volume of distribution of approximately $4.6 \mathrm{~L} / \mathrm{kg}$.

Since voriconazole is eliminated predominantly by metabolism, it is likely that its non-linear pharmacokinetic profile is probably due to saturation of its metabolism with respect to dose. The variability in plasma concentrations and systemic exposure varies between patients, depending on the genotype of the hepatic cytochrome P450 (CYP) enzymes. The CYP2C19 isoenzyme, for which voriconazole is an important substrate, displays frequent genetic polymorphisms [18]. Homozygous extensive metabolizers have a significantly lower exposure to voriconazole than heterozygous extensive or poor metabolizers. Poor metabolizers can have up to 4 times higher serum voriconazole concentrations than extensive metabolizers [19]. However, to date, no dosage adjustments are recommended with regard to these observations.

Overall, the CYP2C19 genotype accounts (in addition to gender and age) for a large variability in clearance and AUC of voriconazole.

\section{DRUG INTERACTIONS}

As voriconazole is a substrate for CYP450 isoenzymes, multiple drug-drug interactions must be considered. Particularly, the CYP2C19 genotype and co- 
Table 2. Drug interactions with voriconazole.-

\begin{tabular}{|c|c|}
\hline Drug & Management \\
\hline \multicolumn{2}{|l|}{ Decreases voriconazole interactions } \\
\hline Rifampin & contraindicated \\
\hline Rifabutin & contraindicated \\
\hline Phenytoin & monitoring \\
\hline Carbamazepine & contraindicated \\
\hline Long-acting barbiturates & contraindicated \\
\hline Ritonavir & contraindicated \\
\hline Efavirenz & contraindicated \\
\hline \multicolumn{2}{|l|}{ Increases in concentration by voriconazole } \\
\hline Sirolimus & contraindicated \\
\hline Rifabutin & contraindicated \\
\hline Efavirenz & contraindiacated \\
\hline Quinidine & contraindicated \\
\hline Tacrolimus & monitoring \\
\hline Cyclosporine & monitoring \\
\hline Omeprazole & reduce dose by half \\
\hline Warfarin & monitoring \\
\hline Methadone & reduce dose \\
\hline \multicolumn{2}{|c|}{ Likely increases in concentration by voriconazole } \\
\hline Ergot alkaloids & contraindicated \\
\hline Pimozide & contraindicated \\
\hline Sulfonylureas & monitoring \\
\hline HMG-CoA reductase inhibitors & monitoring \\
\hline Vinca alkaloids & monitoring \\
\hline Calcium channel blockers & monitoring \\
\hline Benzodiazepines & monitoring \\
\hline Prednisolone & monitoring \\
\hline HIV protease inhibitors, not indinavir & monitoring \\
\hline
\end{tabular}

administration of drugs that modulate its activities may affect voriconazole plasma concentrations (Table 2). Voriconazole serum concentrations are significantly reduced by rifampin, rifabutin, phenytoin as well as carbamazepine and long-acting barbiturates [19]. At the same time, plasma concentrations of rifabutin and phenytoin may be increased resulting in the need of monitoring their serum levels. The metabolism of other drugs that are a substrate of CYP metabolic enzymes may also be influenced by co-administration of voriconazole and can result in clinically relevant toxicity if the therapeutic index is small. The list of contraindicated co-medication includes sirolimus and ergot alkaloids. Dose monitoring of cyclosporine and tacrolimus is mandatory when used in combination with voriconazole, since the latter can increase their serum concentrations. This is also clinically relevant for concomitant administration of HMG-CoA reductase inhibitors, sulfonylureas, vinca alkaloids, calcium channel antagonists and benzodiazepines.

Voriconazole drug-drug interactions are highly clinically relevant, especially in the treatment of patients with leukemia and after stem cell transplantation, since voriconazole may interact with immunosuppressive drugs and chemotherapeutic agents.

\section{PHARMACODYNAMICS}

Pharmacodynamics describes the relationship between drug exposure and outcome (Table 3). Voriconazole is
Table 3. Pharmacodynamic parameters of voriconazole.

\begin{tabular}{ll}
\hline Parameter & Characteristics \\
\hline Activity & $\begin{array}{l}\text { Candida: fungistatic } \\
\text { Aspergillus: fungicidal } \\
\text { Time course of activity } \\
\text { Candida: time-dependent fungistatic } \\
\text { Aspergillus: time-dependent slowly } \\
\text { fungicidal } \\
\text { Candida: no PAFE } \\
\text { Aspergillus: short PAFE }\end{array}$ \\
\hline
\end{tabular}

PAFE, post-antifungal effect

highly effective against most Candida spp. including C. albicans, C. glabrata, C.tropicalis, C.parapsilosis and C. krusei. Resistance to all available azole antifungals may occur in strains of C. glabrata and C.krusei [20]. Using macro- and microdilution test methods for the antifungal susceptibility of filamentous fungi, voriconazole is shown to be highly active against A. fumigatus, with $>95 \%$ of 114 isolates being inhibited at concentrations below $0.5 \mu \mathrm{g} / \mathrm{ml}$ [20]. Voriconazole is moderately active against Fusarium spp. and Scedosporium spp., but shows no relevant activity against Zygomycetes such as Rhizopus, Mucor, Rhizomucor, Cunninghamella or Absidia [21].

Voriconazole is recommended for first-line treatment of invasive aspergillosis in patients with hematologic malignancies and post allogeneic stem cell transplantation [22], since it has been associated with superior response and survival rates in a prospective randomized clinical trial comparing the drug with the former gold standard of amphotericin B deoxycholate [23]. In a post-hoc analysis, it could be demonstrated, that response and survival rates in voriconazole-treated patients were approximately $20 \%$ higher if the antifungal was started on the basis of a typical radiological sign of invasive pulmonary aspergillosis, as compared to a deferred treatment based upon criteria for proven or probable aspergillosis, as defined by a consensus panel of the European Organization for Research and Treatment of Cancer and the Mycoses Study Group of the National Cancer Institute. The place of voriconazole in the treatment algorithm of empiric therapy for antibiotic-refractory neutropenic fever is less well defined. A prospective study of voriconazole versus liposomal amphotericin B has shown a very low rate of breakthrough fungal infections in voriconazole recipients in this setting, but failed non-inferiority in the primary composite endpoint and thereby the approval of voriconazole for empirical antifungal therapy in febrile neutropenic patients. Further studies of voriconazole in this clinical scenario are ongoing. Voriconazole has demonstrated non-inferiority to fluconazole in prophylaxis of invasive fungal disease in patients after allogeneic stem cell transplantation in a recently published phase III trial. When compared to fluconazole $400 \mathrm{mg}$ daily, it reduced the incidence of invasive aspergillosis, but was non-superior with respect to disease-free survival or overall survival in this patient population at the pre-defined primary endpoint, i.e. 180 days posttransplant [24].

The efficacy of voriconazole may be related to its serum concentration. While this has never been 
demonstrated in a prospective phase III trial, retrospective data suggest that it might be reasonable to maintain serum voriconazole trough concentrations of 1-2 mg/1 to ensure efficacy. The value of a regular and routine therapeutic drug monitoring, however, is still debated [25].

\section{POSACONAZOLE}

Posaconazole is a lipophilic triazole antifungal agent with broad-spectrum antifungal activity in vitro and in vivo against most Candida spp., Cryptococcus neoformans, Aspergillus spp. and several zygomycetes [26]. It has been developed for prophylaxis and treatment of fungal infections in immunocompromised individuals with special consideration of patients with hematologic malignancies and allogeneic stem cell transplant recipients. Posaconazole is the first antifungal drug that has demonstrated superior overall survival when used for antifungal prophylaxis in patients with acute myeloid leukemia or myelodysplastic syndrome undergoing intensive remission induction chemotherapy.

\section{PHARMACOKINETICS}

Posaconazole is available as an oral formulation. Its pharmacokinetic profile is characterized by a strong impact of nutrients on its absorption, a long elimination half-life and, in contrast to voriconazole, an only minor metabolism via cytochrome P450 isoenzymes [27]. The oral absorption of posaconazole is dose-limited and strongly dependent on the intake of fat-containing food. The time to reach the maximum plasma concentration is 5-8 hours after administration of a single dose [28]. The relative bioavailability is significantly increased by administration in divided doses. Compared with a single dose, the relative bioavailability of posaconazole $800 \mathrm{mg}$ is increased by $98 \%$ when administered in two divided doses every 12 hours, and by $220 \%$ when divided in 4 doses administered at 6hours intervals [29]. Studies on plasma concentrations with doses between $50 \mathrm{mg}$ and $800 \mathrm{mg}$ showed a saturation of absorption occurring above $800 \mathrm{mg}$. In febrile neutropenic patients or those with refractory invasive fungal disease, there was no further increase in exposure when the dose was increased from $400 \mathrm{mg}$ to $600 \mathrm{mg}$ twice daily [30].

Posaconazole is highly protein bound $(>98 \%)$ and the binding is concentration-independent. This lipophilic drug has a large volume of distribution at steady-state from approximately $5 \mathrm{~L} / \mathrm{kg}$ to $25 \mathrm{~L} / \mathrm{kg}$. This suggests extensive extravascular distribution and penetration into intracellular spaces. Steady-state concentrations are achieved after 7-10 days resulting in a mean terminal half-life of around 35 hours [27]. Peak serum concentration differs significantly between individuals although differences in renal or hepatic function, gender, or body mass or surface area differences do not account for this variation. The reasons for this interindividual variability remain poorly defined.

Posaconazole is not metabolized to a significant extent through the CYP enzyme system [31]. The metabolism of posaconazole is mediated predominantly through phase-2 biotransformation via uridine diphos- phate (UDP)-glucuronyltransferase (UGT) enzyme pathways [32]. Posaconazole is also both a substrate and an inhibitor of p-glycoprotein. The role of polymorphisms in genes encoding drug-metabolizing enzymes and transporters in the pharmacokinetics of posaconazole remains to be explored in detail.

Oral food intake increases the bioavailability of posaconazole [33]. Systemic exposure increases by 4and 2.6-fold when it is consumed with a high-fat or non-fat meal, respectively, when compared to fasting individuals. Administering posaconazole with non-fat food increases its exposure by $164 \%$ while inclusion of fat in a meal further increases posaconazole exposure by $48 \%$. Therefore, posaconazole should be administered with a full meal whenever possible, in order to optimize absorption. In patients who are unable to eat, e.g. those with severe mucositis after stem cell transplantation, the addition of a liquid nutritional supplement to the administration of posaconazole has shown efficacy in maintaining sufficient absorption of the drug [34].

For prophylaxis of IFD in patients with acute leukaemia or with GvHD after allogeneic stem cell transplantation, posaconazole is administered at a dosage of $600 \mathrm{mg}$ daily divided in 3 doses, and for systemic treatment in patients with invasive fungal disease, $800 \mathrm{mg}$ daily divided in 2 doses with a full meal or 4 doses without meal.

\section{DRUG INTERACTIONS}

The azole drug-drug interactions include competition for absorption and metabolism, resulting in altered serum concentrations of posaconazole itself or its counterpart. As posaconazole undergoes hepatic metabolism via UDP glucuronidation and via p-glycoprotein efflux, inhibitors or inducers of these clearance pathways may effect posaconazole serum concentrations. As with other azole antifungals, in vitro studies have shown that posaconazole is an inhibitor primarily of CYP3A4. Plasma concentration of drugs predominantly metabolized by this pathway may be altered by posaconazole. A summary of possible drug interactions is presented in Table 4.

\section{PHARMACODYNAMICS}

Pharmacodynamics refers to the time course and intensity of drug effects on an organism. In these analyses, both the Cmax and the AUC are important factors in determining the efficacy of posaconazole in experimental settings. Posaconazole has shown activity against fungal infections including invasive candidiasis, aspergillosis, cryptococcosis, histoplasmosis, coccidioidomycosis, fusariosis and mucormycosis in numerous preclinical animal and human models [26].

Posaconazole has considerable in vivo antifungal activity, which has been demonstrated in clinical trials. In clinical practice, it is the drug of first choice for antifungal prophylaxis in patients with acute myeloid leukemia or myelodysplastic syndrome undergoing intensive remission induction chemotherapy as well as in patients with graft-versus-host disease requiring immunosuppressive treatment [22]. 
Table 4. Drug interactions with Posaconazole.

\begin{tabular}{ll}
\hline Drug & Management \\
\hline Concentration increased by posaconazole \\
Atazanavir & monitoring \\
Cyclosporine & reduce dose \\
Lorazepam & contraindicated \\
Midazolam & reduce dose \\
Sirolimus & contraindicated \\
Tacrolimus & reduce dose
\end{tabular}

Concentration is increased by posaconazole/decreases posaconazole concentration

Phenytoin

avoid co-administration

Rifabutin avoid co-administration

Decreases posaconazole concentration

Cimetidine

avoid co-administration

Efavirenz avoid co-administration

Concentration may be increased by posaconazole

Alprazolam

Amiodarone

Astemizole

Benzodiazepines

Calcium channel blockers

Carbamazepine

Cisapride

Corticosteroid

Digoxin

Drugs for erectile dysfunction

(e.g. sildenafil)

Ergot alkaloids

HMG-CoA reductase inhibitors

Irinotecan

Quinidine

Sulfonylureas

Terfenadine

Vinca alkaloids

reduce dose

monitoring

contraindicated

monitoring

monitoring

monitoring

contraindicated

monitoring

monitoring

monitoring

contraindicated

monitoring

monitoring

contraindicated

monitoring

reduce dose

monitoring

May decrease posaconazole concentrations

Famotidine

avoid co-administration

Omeprazole

Ranitidine

Rifampin

avoid co-administration

avoid co-administration monitoring

Ullmann et al. randomized over 500 patients with graft-versus-host disease undergoing intensive immunosuppressive therapy to antifungal prophylaxis with either posaconazole or fluconazole [35]. The incidence of proven or probable invasive fungal infection was $5.3 \%$ versus $9 \%$, thus not reaching statistically significant difference. A significant reduction in the number of cases of invasive aspergillosis in posaconazole recipients was recorded. While all-cause mortality was not reduced, a significant difference was observed with respect to mortality due to invasive fungal infection $(1 \%$ versus $4 \%)$. The number needed to treat (NNT) in order to prevent one invasive fungal infection with the use of posaconazole versus fluconazole was 27, whereas the NNT to prevent 1 case of invasive aspergillosis was 22 .

Cornely et al. studied the role of posaconazole for prophylaxis of IFD in patients with acute leukemia or myelodysplastic syndrome undergoing intensive chemotherapy in a phase III trial comparing posacona- zole with standard azole prophylaxis in 602 patients [36]. Patients were randomized to receive either posaconazole or fluconazole or itraconazole, with the latter selected by local investigators. The mean duration of prophylaxis was 29 days in the posaconazole patients and 25 days in the comparator group. Posaconazole significantly reduced the incidence of IFD from $8 \%$ to $2 \%$, providing effective prevention of invasive aspergillosis. For the first time, posaconazole improved overall survival in this patient population. The NNT to prevent 1 invasive fungal infection or 1 case of aspergillosis was 17 for each. The NNT to prevent one death was 33. Prophylaxis failures were not explained by the variability in bioavailability of posaconazole. Plasma concentrations of the drug in patients with breakthrough fungal infection were not significantly different from those in the overall patient population.

These two large randomized trials have established the role of posaconazole as antifungal of choice for prophylaxis in these specific patient populations [37]. Although there is no head-to-head randomized trial to date, posaconazole appears superior to voriconazole for prophylaxis of IFD in patients with acute leukemia or myelodysplastic syndrome. Current evidence-based guidelines recommend its prophylactic use in this high-risk patient population undergoing intensive myelosuppressive chemotherapy.

Posaconazole has been evaluated as salvage therapy in patients with refractory IFD [38]. The partial or complete response rate was $60 \%$ among patients who had treatment failure with or were intolerant of other antifungal drugs. One-fifth of these patients had developed breakthrough infections with a zygomycete while receiving voriconazole. In this patient cohort, $62 \%$ survived and around $40 \%$ of deaths were attributed to fungal infection. In another study in patients with refractory invasive aspergillosis in 107 patients, success was obtained in $42 \%$ of patients treated with posaconazole [39]. Successful outcome was associated with higher serum concentrations of posaconazole. In a quartile analysis of steady-state maximum or average plasma concentration and response, the response rate in those $25 \%$ patients with lowest concentrations was $24 \%$ in opposite to the quartile of patients with highest concentrations, in whom a $75 \%$ success rate could be demonstrated [39].

The therapeutic value of serum level monitoring of posaconazole remains unclear to date. In a subsequent analysis of the two above-mentioned posaconazole prophylaxis trials, posaconazole concentrations were found to significantly correlate with a composite efficacy end point including parameters such as absence of premature discontinuation of the prophylactic drug, no IFD and no death. However, the major driving factor for clinical failure was the administration of empiric antifungal therapy and not breakthrough fungal infections or death. Based upon these data, posaconazole drug monitoring cannot be recommended by now.

\section{REFERENCES}

1. Marr KA, Carter RA, Crippa $F$ et al. Epidemiology and outcome of mould infections in haematopoietic stem cell recipients. Clin Infect Dis 2002; 34:909-17 
2. Pagano L, Caira M, Candoni A et al. The epidemiology of fungal infections in patients with hematological malignancies: the SEIFEM-2004 study. Haematologica 2006; 91: 1068-75

3. Cuenca-Estrella M, Bernal-Martinez I, Buitrago MJ et al. Update on the epidemiology and diagnosis of invasive fungal infections. Int J Antimicrob Agents 2008; 32(suppl2): S 143-7

4. Patterson TF. Advances and challenges in management of invasive mycosis. Lancet 2005; 366: 1013-25

5. Steinbach WJ, Stevens DA. Review of newer antifungal and immunomodulatory strategies for invasive aspergillosis. Clin Infect Dis 2003: 37(suppl3): S 157-87

6. Verweij PE, Mellado E, Melchers WJ. Multiple-triazole-resistant aspergillosis. N Engl J Med 2007; 356: 1481-3

7. Boucher HW, Groll AH, Chiou CC et al. Newer systemic antifungal agents: pharmacokinetics, safety and efficacy. Drugs 2004; 64: 1997-20

8. Purkins L, Wood N, Greenhalgh K et al. Voriconazole, a novel wide-spectrum triazole: oral pharmacokinetics and safety. Br J Clin Pharmacol 2003; 56 Suppl 1: 10-6

9. Purkins L, Wood N, Kleinermans D, Greenhalgh K et al. Effect of food on the pharmacokinetics of multiple-dose oral Voriconazole. Br J Clin Pharmacol 2003; 56: 17-23

10. Purkins L, Wood N, Ghahramani P et al. Pharmacokinetics and safety of Voriconazole following intravenous- to oraldose escalation regimens. Antimicrob Agents Chemother 2002; 46: 2546-53

11. Hyland R, Jones BC, Smith DA. Identification of the cytochrome P450 enzymes involved in the N-oxidation of Voriconazole. Drug Metab Dispos 2003; 31: 540-7

12. Miyakis S, van Hal SJ, Ray J et al. Voriconazole concentrations and outcome of invasive fungal infections. Clin Infect Dis 2010; 16: 927-33

13. Bruggemann RJ, Blijlevens NM, Burger DM et al. Pharmacokinetics and safety of 14 days intravenous voriconazole in allogeneic haematopoietic stem cell transplant recipients. J Antimicrob Chemother 2010; 65: 107-13

14. Denning DW, Ribaud P, Milpied N et al. Efficacy and safety of voriconazol in the treatment of acute invasive aspergillosis. Clin Infect Dis 2002; 34: 563-71

15. Trifilio S, Pennick G, Pi J et al. Monitoring plasma voriconazole levels may be necessary to avoid subtherapeutic levels in haematopoietic stem cell recipients. Cancer 2007; 109: 1532-5

16. Craig WA, Suh B. Protein binding and the antimicrobial effects: methods for determination of protein binding. In: Lorian V, editor. Antibiotics in laboratory medicine. Philadelphia (PA): Lippincott Williams \& Wilkins 1991: 367-402.

17. Jeu L, Piacenti FJ, Lyakhovetskiy AG et al. Voriconazole. Clin Ther 2003; 25: 1321-81

18. Ikeda Y, Umemura K, Kondo K et al. Pharmacokinetics of Voriconazole and cytochrome P450 2C19 genetic status. Clin Pharmacol Ther 2004; 75: 587-8

19. Pfizer Inc. Label: Voriconazole for injections, tablets, oral suspension: LAB -0271-12; 2005 Mar

20. Drago M, Scaltrito MM, Morace G. In vitro activity of voriconazole and other antifungal agents against clinical isolates of Candida glabrata and Candida krusei. Eur J Clin Microbiol Infect Dis 2004; 23: 619-24

21. Gil-Lamaignere C, Hess R, Salvenmoser S. Effect of media composition and in vitro activity of Posaconazole, Caspofungin and Voriconazole against zygomycetes. J Antimicrob Chemother 2005; 55: 1016-9

22. Walsh TJ, Anaissie EL, Denning DW et al. Treatment of Aspergillosis: clinical practice guidelines of the Infectious Diseases Society of America. Clin Infect Dis 2008; 46: 327 60

23. Herbrecht R, Denning DW, Patterson TF et al., Voriconazole versus amphotericin B for primary therapy of invasive aspergillosis. N Engl J Med 2002; 347: 408-15
24. Wingard JL, Carter SL, Walsh TJ et al. Randomized, double-blind trial of fluconazole versus voriconazole for prevention of invasive fungal infection after allogeneic haematopoietic cell transplantation. Blood 2010; 116: 511118

25. Smith JA. What is the role of therapeutic drug monitoring in antifungal therapy? Curr Infect Dis Rep 2009; 11:439-46

26. Nagappan V, Deresinski S. Posaconazole: a broad-spectrum triazole antifungal agent. Clin Infect Dis 2007; 45: 1610-7

27. Li Y, Theuretzbacher U, Clancy CJ et al. Pharmacokinetic/Pharmacodynamic profile of Posaconazole. Clin Pharmacokinet 2010 ; 49: 379-96

28. Courtney R, Pai S, Laughlin M et al. Pharmacokinetics, safety, and tolerability of oral Posaconazole administered in single and multiple doses in healthy adults. Antimicrob Agents Chemother 2003; 47: 2788-95

29. Ezzet F, Wexler D, Courtney R et al. Oral bioavailability of Posaconazole in fasted healthy subjects: comparison between three regimens and basis for clinical dosage recommendations. Clin Pharmacokinet 2005; 44: 211-20

30. Schering-Plough Inc. Label: Noxafil ${ }^{\circledR}$ (Posaconazole) oral suspension. Labeling - 31029708. Kenilworth (NJ): Schering-Plough Inc., 2006 Oct

31. Wexler D, Courtney R, Richards W et al. Effect of Posaconazole on cytochrome P450 enzymes: a randomized, open-label, two-way crossover study. Eur J Pharm Sci 2004; 21: 645-53

32. Ghosal A, Hapangama N, Yuan Y et al. Identification of human UDP-glucuronosyltransferase enzyme(s) responsible for the glucuronidation of posaconazole (Noxafil $\mathbb{R}$ ). Drug Metab Dispos 2004; 32: 267-71

33. Courtney R, Wexler D, Radwanski E et al. Effect of food on the relative bioavailability of two oral formulations of Posaconazole in healthy adults. Br J Clin Pharmacol 2004; 57: 218-22

34. Courtney R, Radwanski E, Lim J et al. Pharmacokinetics of Posaconazole coadministered with antacid in fasting or nonfasting healthy men. Antimicrob Agents Chemother 2004; 48: 804-8

35. Ullmann A, Lipton J, Vesole D et al. Posaconazole or fluconazole for prophylaxis in severe graft-versus-host disease. N Engl J Med 2007; 356: 335-47

36. Cornely OA, Maertens J, Winston D et al. Posaconazole vs fluconazole or itraconazole prophylaxis for patients with neutropenia. N Engl J Med 2007; 356: 348-359

37. De Pauw BE, Donnelly JP. Prophylaxis and aspergillosis has the principle been proven? N Engl J Med 2007; 356: 409-11

38. van Burik JAH, Hare RS, Solomon HF et al. Posaconazole is effective as salvage therapy in zygomycosis: a retrospective summary of 91 cases. Clin Infect Dis 2006: 42:e61-5

39. Walsh TJ, Raad I, Patterson TF et al. Treatment of invasive aspergillosis with posaconazole in patients who are refractory to or intolerant of conventional therapy: an externally controlled trail. Clin Infect Dis 2007; 44: 2-12

Received: February 7, 2011 / Accepted: February 22, 2011

Address for correspondence:

Georg Maschmeyer, MD

Klinikum Ernst von Bergmann

Dept. of Hematology, Oncology and Palliative Care

Charlottenstrasse 72

D-14467 Potsdam

Germany

E-mail: gmaschmeyer@klinikumevb.de

Michael Sandherr, MD

Röntgenstr. 4/II

D-82362 Weilheim

Germany 